United Nations Educational Scientific and Cultural Organization

and

International Atomic Energy Agency

THE ABDUS SALAM INTERNATIONAL CENTRE FOR THEORETICAL PHYSICS

\title{
SELF-CONSISTENT NONLINEARLY POLARIZABLE SHELL-MODEL DYNAMICS FOR FERROELECTRIC MATERIALS
}

\author{
S.E. Mkam Tchouobiap \\ Laboratoire de Mécanique, Département de Physique, Faculté des Sciences, \\ Université de Yaoundé I, BP 812, Yaoundé, Cameroun, \\ C.M. Ngabireng ${ }^{1}$ \\ Departement de Mathematiques et de Sciences Physiques, Ecole Nationale Supérieure Polytechnique, \\ Université de Yaoundé I, BP 8390, Yaoundé, Cameroun \\ and \\ The Abdus Salam International Centre for Theoretical Physics, Trieste, Italy \\ and \\ T.C. Kofane \\ Laboratoire de Mécanique, Département de Physique, Faculté des Sciences, \\ Université de Yaoundé I, BP 812, Yaoundé, Cameroun.
}

\begin{abstract}
We investigate the dynamical properties of the polarizable shell-model with a symmetric double Morse-type electron-ion interaction in one ionic species. A variational calculation based on the Self-Consistent Einstein Model (SCEM) shows that a theoretical ferroelectric (FE) transition temperature can be derived which demonstrates the presence of a first-order phase transition for the potassium selenate $\left(\mathrm{K}_{2} \mathrm{SeO}_{4}\right)$ crystal around $\mathrm{Tc}=91.5 \mathrm{~K}$. Comparison of the model calculation with the experimental critical temperature yields satisfactory agreement.
\end{abstract}

MIRAMARE - TRIESTE

November 2002

\footnotetext{
${ }^{1}$ Regular Associate of the Abdus Salam ICTP.
} 


\section{I- Introduction}

There have been several ways of describing lattice dynamics of ferroelectricity: (i) The soft mode concept and the conventional anharmonic perturbation techniques. The idea which is of critical importance here is that for a crystal which undergoes a lattice structural transformation from a hightemperature paraelectric phase to a low-temperature ordered ferroelectric phase, the harmonic approximation represents an unstable state of equilibrium and the crystal will be characterized by imaginary harmonic frequencies for a group of optic modes of long wavelength $[1,2]$. (ii) Order parameter description of the ferroelectric crystal. The concept of the order parameter was originally introduced by Landau [3]. When used to describe lattice structural transformations, the order parameter denotes the degree by which the equilibrium ionic configuration of the less symmetrical (ordered) phase deviates from the ionic configuration of the more symmetrical (disordered) phase. In the disordered phase the order parameter is identically zero, whereas it takes on a non-zero value in the ordered phase, with the transition between the two phases being either continuous (2nd order) or discontinuous (1st order). (iii) Variational treatment of a model ferroelectric. A variational approach is of particular interest from the point of view that it provides a bound on the free energy and at the same time assures that the model crystal occupies a stable state of equilibrium. The first detailed calculations on a model system employing a variational technique were first carried out by Gillis and Koehler [4.5], who determined self-consistently the frequency spectrum of a model crystal exhibiting a displacive transition from a high-temperature $\mathrm{NaCl}$ structure phase to a low-temperature distorted rhombohedral phase. The idea which underlies all of the variational approaches is simply that if the low-lying collective excitation spectrum of the crystal is well described by phonons, then one can introduce a trial harmonic Hamiltonian to describe the dynamics of these excitations, with the parameters in the Hamiltonian determined variationally. (iv) Mean field theory. In this approach, the excitation spectrum of the crystal is viewed as a collection of dynamically coupled normal modes of phonon-like character. The self- consistent feature arises from the fact that in an actual crystal lattice each atom feels not the bare potential of the remaining atoms, as in the harmonic approximation, but rather an effective potential which incorporates in some manner an average over the mean positions of all the particles, each of which is treated equivalently [6-11]. (v) One-phonon response function. In ferroelectrics where soft phonon mode behavior is an integral feature of the structural transformation, the inelastic neutron technique provides an important tool for determining the temperature dependence and symmetry of the soft mode both above and below the transition [12].

In the present paper, we give an analytical description of the ferroelectric phase transition in a nonlinear discrete polarizability model where oxygen $\left(\mathrm{O}^{2-}\right)$ or chalcogen $\left(\mathrm{S}^{2-}\right.$, etc. $)$ ions play a central role. In general, the microscopic origin of the ferroelectric phase transition may be found in the intrinsic electronic instability of the $\left(\mathrm{O}^{2-}\right)$ ion and its homologues $\left(\mathrm{S}^{2-}\right)$, which in ionic compounds are stabilized by long-range coulomb forces only [13]. In our shell-model, the lowest term of the nonlinear 
core-shell interaction at the oxygen or chalcogen site is of the double-well potential resulting from a combination of two Morse potentials.

The organization of the paper is as follows. In section II, we present the polarizability model Hamiltonian. In section III, the description of the ferroelectric phase transition is carried out on the basis of the SCEM. The relative displacement and the two trial parameters representing Einstein frequencies of the core and shell atoms respectively are calculated in the general case. The critical ferroelectric phase transition temperature is estimated in section IV in two principal directions: Firstly, when the shell-atom fluctuations are neglected, and secondly in the high-temperature regime. In section V, we consider the model Hamiltonian for certain special cases, namely the core and shell masses are identical, and the harmonic nearest-neighbour interactions shell-shell, core-core are also identical. The analytical expression of the critical ferroelectric phase transition temperature is obtained. Numerical values are estimated for the critical first-order phase transition temperature for the $\mathrm{K}_{2} \mathrm{SeO}_{4}$ crystal in section VI. Section VII concludes the paper.

\section{II- The polarizability model Hamiltonian}

The model under consideration consists of a monatomic chain of nonlinearly polarizable atoms with harmonic nearest-neighbour interactions, shell-shell $\mathrm{f}$ and core-core $\mathrm{f}$ ', and double-well local core-shell interaction as shown in Fig.1. Two neighboring polarizable atoms are connected by harmonic potentials (core-core and shell-shell) to keep the model as simple as possible. Also, the bonds connecting the core and shell atoms belonging to the same atoms are extremely stretched so that their nonlinearity must not be ignored. Usually, the above double-well local core-shell interaction is chosen in the following quartic form $[13,14]$

$$
V\left(w_{i}\right)=\frac{1}{2} g_{2} w_{\mathrm{i}}^{2}+\frac{1}{4} g_{4} w_{\mathrm{i}}^{4}
$$

where $\mathrm{g}_{2}<0, \mathrm{~g}_{4}>0$, and $w_{\mathrm{i}}=v_{\mathrm{i}}-u_{\mathrm{i}}$ is the relative displacement, $v_{\mathrm{i}}$ denotes the shell displacement while $u_{i}$ denotes the core displacement of the ith atom. The double-well potential (1) combines harmonic attractive electron-phonon interaction $\mathrm{g}_{2}$ with a stabilizing repulsive electron-two-phonon coupling term $\mathrm{g}_{4}$. The microscopic reasoning for this potential is due to the local instability of the oxygen ion $\mathrm{O}^{2-}$ that leads to a volume-dependent and temperature-dependent nonlinear polarizability [13]. Although the physical origin of the double-well potential is well-known, its modelisation by a polynomial function of quartic-type is a severe approximation since minima of this double-well potential as well as the height of its barrier are fixed. For instance, phase transitions in ferroelectric systems are usually connected only with the rearrangements of a few atoms in the unit cell whereas the positions of all others remain unchanged. Examples are the displacements of the Ti ions with respect to the $\mathrm{O}_{6}$ octahedra in $\mathrm{BaTiO}_{3}$, the rotations of the $\mathrm{O}_{6}$ octahedra in $\mathrm{SrTiO}_{3}$, the rearrangement of the 
protons in the double-well potentials of the $\mathrm{O}-\mathrm{H}---\mathrm{O}$ hydrogen bonds in hydrogen bonded ferroelectrics. In those systems, the shape of the nonlinear one-site potential may deviate considerably from that attributed to the local potential, as in the hydrogen-bonded system where the large displacement of the heavy ions can significantly modify the barrier height of the double-well potential associated with the light proton. In this paper, we present some results with the double-well potential $\mathrm{V}\left(w_{i}\right)$ of the double Morse-type potential. As depicted in Fig. 1 the expression of $\mathrm{V}\left(w_{i}\right)$ is then

$$
\mathrm{V}\left(\mathrm{w}_{i}\right)=\mathrm{V}_{0}\left\{\frac{\mathrm{A}^{2}}{4}\left[\exp \left(2 \alpha w_{i}\right)+\exp \left(-2 \alpha w_{i}\right)\right]-\mathrm{A}\left[\exp \left(\alpha w_{i}\right)+\exp \left(-\alpha w_{i}\right)\right]\right\}
$$

where $\mathrm{V}_{0}, \mathrm{~A}$ and $\alpha$ are the positive parameters of the potential. The parameter $\mathrm{A}$ with $0<\mathrm{A}<1$ is given by the formula [15]

$$
\mathrm{A}=2 \exp \left\{-\alpha\left[\frac{R}{2}-r_{0}\right]\right\}
$$

where $\mathrm{R}$ is the distance between the two sublattices (each core and shell atoms); $\alpha$ and $\mathrm{r}_{0}$ are the Morse phenomenological parameters. The constant $\alpha^{-1}$ fixes the length scale over which the nonlinearity of the potential shows up, while $\mathrm{V}_{0}$, which is the dissociation energy, sets the energy scale. The value $\Delta \mathrm{V}=\mathrm{V}_{0}\left(1-\mathrm{A}^{2}\right)$ corresponds to the height of the potential barrier between two minima of the potential given by $\pm w_{0}$, with $w_{0}=(1 / \alpha) \operatorname{arccosh}(1 / \mathrm{A})$. Figure 1 , which gives the shape of the potential $\mathrm{V}\left(w_{i}\right)$ for different values of $\mathrm{A}$, shows that as A increases the barrier height of the doublewell continuously decreases. On the other hand, the minima of the potential vary continuously with A. The idea that the double-well potential can be constructed by placing back to back two Morse potentials goes back to Lawrence and Robertson [16] and Matsushita and Matsubara [17] in the theory of hydrogen bonded ferroelectrics. Various models based on the same idea [15,18-20] have followed these pioneering works. These two Morse potentials have been introduced later by other authors in the context of soliton theories [21-24]. An extension which attempts to combine both dispersion and nonlinearity. Their balance is responsible for the existence of the solitons. The total Hamiltonian $\mathrm{H}$ of the model can be expressed as

$$
\mathrm{H}=\mathrm{T}+\mathrm{U},
$$

where the kinetic and potential energies are respectively given by

$$
\begin{aligned}
& T=\frac{1}{2 M} \sum_{i} P_{i}^{2}+\frac{1}{2 m} \sum_{i} p_{i}^{2}, \\
& U=\sum_{i}\left[\frac{1}{2} f^{\prime}\left(u_{i+1}-u_{\mathrm{i}}\right)^{2}+\frac{1}{2} f\left(v_{i+1}-v_{i}\right)^{2}+V\left(v_{i}-u_{i}\right)\right],
\end{aligned}
$$

$\mathrm{M}$ and $\mathrm{m}$ are core and shell masses, $\mathrm{P}_{\mathrm{i}}$ denotes a core momentum of the ith atom of mass $\mathrm{M}$, and $\mathrm{p}_{\mathrm{i}}$, the shell momentum of the ith atom of mass $\mathrm{m}$. The overdot (.) indicates the time derivative. 


\section{III- Self-Consistent Einstein Model}

In this section, we shall investigate the dynamical properties of ferroelectric phase transition systems with a symmetric double Morse-type potential by using the Self-Consistent Einstein Model in which the theoretical treatment also take into account the quantum nature of the shell and core vibrations.

The basis of SCEM is a variational relation for free energy of the system and most be easily formulated without resorting to a normal mode decomposition. Indeed, we define a trial Hamiltonian which is harmonic in form and then constructs a displaced oscillator trial crystal density matrix, which is quasiharmonic in form and depends on a set of variational force constants, based on this trial Hamiltonian and produced excellent approximations to thermodynamic quantities.

We first assume that the system is well described by the trial Hamiltonian

$$
H_{0}=\frac{1}{2 M} \sum_{i}^{N} P_{i}^{2}+\frac{K}{2} \sum_{i=1}^{N}\left(\delta u_{i}\right)^{2}+\frac{1}{2 m} \sum_{i=1}^{N} p_{i}^{2}+\frac{k}{2} \sum_{i=1}^{N}\left(\delta v_{i}\right)^{2}
$$

represented in terms of the harmonic Einstein oscillators in SCEM [18], where $\delta u_{i}$ is the vibrational displacement of ith core and $\delta v_{i}$ is that of ith shell atoms about their equilibrium positions. The parameters $\mathrm{K}$ and $\mathrm{k}$ are the trial force constants that may be determined by minimizing the trial variational free energy $F_{v}$ with respect to variations in $K$ and $k$.

We begin by assuming the trial density matrix of the entire crystal to have the form $[25,26]$

$$
\rho_{\mathrm{c}}=\frac{e^{-\beta H_{0}}}{Z_{0}}
$$

where $Z_{0}$ is the partition function for the trial Hamiltonian defined by

$$
Z_{0}=\operatorname{Tr}\left(e^{-\beta H_{0}}\right),
$$

where $\beta=1 / k_{B} T, k_{B}$ being the Boltzmann constant, $T$ the temperature.

With a trial Hamiltonian $\mathrm{H}_{0}$ and a corresponding set of states, the variational free energy for SCEM is defined by

$$
F_{v}=\operatorname{Tr}\left\{\rho_{c}\left[H+\beta^{-1} \ln \rho_{c}\right]\right\}
$$

Using (8) and (9) one has the new expression of variational free energy in the form

$$
\begin{aligned}
\mathrm{F}_{\mathrm{v}}=\langle\mathrm{H}\rangle-\left\langle\mathrm{H}_{0}\right\rangle+\mathrm{F}_{0} \\
=\langle U\rangle-\frac{\mathrm{K}}{2} \sum_{i=1}^{N}\left\langle\left(\delta u_{i}\right)^{2}\right\rangle-\frac{k}{2} \sum_{i=1}^{N}\left\langle\left(\delta v_{i}\right)^{2}\right\rangle+F_{0},
\end{aligned}
$$

where $\langle\ldots\rangle$ indicates the thermal average with respect to the trial harmonic Hamiltonian defined by 


$$
\langle\ldots\rangle=\frac{\operatorname{Tr}\left[(\ldots) \exp \left(-\beta H_{0}\right)\right]}{\operatorname{Tr}\left[\exp \left(-\beta H_{0}\right)\right]}
$$

and

$$
\mathrm{F}_{0}=-\beta^{-1} \ln \operatorname{Tr}\left(\mathrm{e}^{-\beta \mathrm{H}_{0}}\right),
$$

the free energy corresponding to $\mathrm{H}_{0}$.

It can be shown [27-29] that $F_{v}$ provides an upper bound to the free energy corresponding to the original Hamiltonian. With the aid of Eq.(6), one may make use of the familiar properties of harmonic lattice dynamics to evaluate $F_{0}$ as

$$
\mathrm{F}_{0}=\mathrm{Nk}_{\mathrm{B}} \mathrm{T}\left\{\ln \left[2 \sinh \left(\frac{\hbar \mathrm{W}}{2 \mathrm{k}_{\mathrm{B}} \mathrm{T}}\right)\right]+\ln \left[2 \sinh \left(\frac{\hbar \omega}{2 \mathrm{k}_{\mathrm{B}} \mathrm{T}}\right)\right]\right\}
$$

with

$$
\begin{aligned}
\mathrm{W} & =\sqrt{\frac{K}{M}}, \\
\omega & =\sqrt{\frac{k}{m}}
\end{aligned}
$$

and where $\mathrm{N}$ is the number of atoms cell.

$\mathrm{W}$ and $\omega$ which are new trial parameters taking the places of $\mathrm{K}$ and $\mathrm{k}$, represent Einstein frequencies of a core atom and a shell atom, respectively which are related to the mean-square fluctuations of a core $\left\langle\left(\delta u_{i}\right)^{2}\right\rangle$ atom and of a shell $\left\langle\left(\delta v_{i}\right)^{2}\right\rangle$ atom as follows[18,26]:

$$
\begin{aligned}
& \frac{K\left\langle\left(\delta u_{i}\right)^{2}\right\rangle}{2}=\frac{\hbar W}{4} \operatorname{coth}\left[\frac{\hbar W}{2 k_{B} T}\right] \\
& \frac{k\left\langle\left(\delta v_{i}\right)^{2}\right\rangle}{2}=\frac{\hbar \omega}{4} \operatorname{coth}\left[\frac{\hbar \omega}{2 k_{B} T}\right]
\end{aligned}
$$

where

$$
\begin{aligned}
& \delta u_{i}=u_{i}-\left\langle u_{i}\right\rangle \\
& \delta v_{i}=v_{i}-\left\langle v_{i}\right\rangle
\end{aligned}
$$

$\left\langle v_{i}\right\rangle$ and $\left\langle u_{i}\right\rangle$ are mean displacements of shell atom and core atom respectively. It is to be note that because of translational invariance the quantities $\left\langle v_{i}\right\rangle,\left\langle u_{i}\right\rangle,\left\langle\left(\delta u_{i}\right)^{2}\right\rangle$ and $\left\langle\left(\delta v_{i}\right)^{2}\right\rangle$ are 
independent on i. Hence, in the future, the reference to the lattice site will be suppressed; that is $\left\langle\left(\delta u_{i}\right)^{2}\right\rangle=\left\langle(\delta u)^{2}\right\rangle,\left\langle\left(\delta v_{i}\right)^{2}\right\rangle=\left\langle(\delta v)^{2}\right\rangle,\left\langle v_{i}\right\rangle=\langle v\rangle$ and $\left\langle u_{i}\right\rangle=\langle u\rangle$.

Calculation of the thermally averaged potential $\langle\mathrm{U}\rangle$ is particularly tedious and so we obtain (see Appendix)

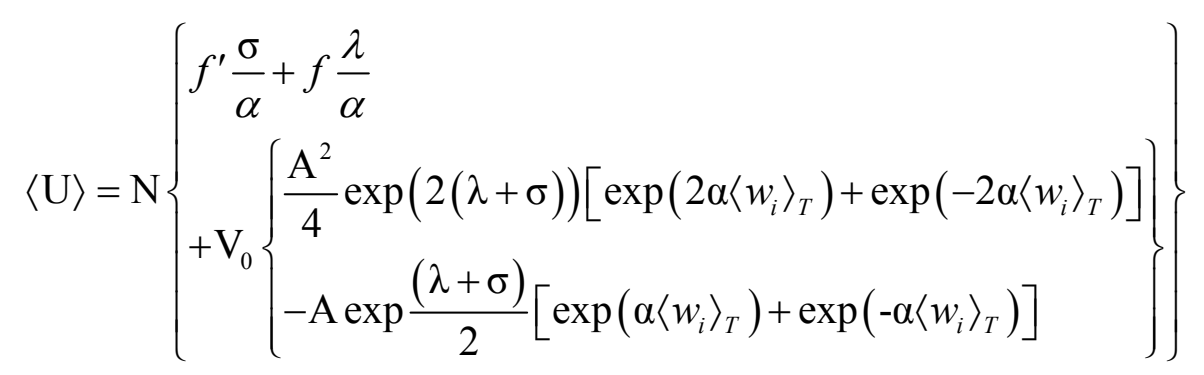

where, $\left\langle w_{i}\right\rangle_{T}=\langle w\rangle_{T}$ is the thermal equilibrium relative displacement between core and shell atoms, and $\lambda$ and $\sigma$ are the fluctuations of shell and core atoms respectively. $\lambda$ and $\sigma$ are defined by

$$
\begin{aligned}
& \lambda=\alpha^{2}\left\langle(\delta v)^{2}\right\rangle=\frac{\hbar \alpha^{2}}{2 \mathrm{~m} \omega} \operatorname{coth}\left(\frac{\hbar \omega}{2 \mathrm{~K}_{\mathrm{B}} \mathrm{T}}\right) \\
& \sigma=\alpha^{2}\left\langle(\delta u)^{2}\right\rangle=\frac{\hbar \alpha^{2}}{2 M W} \operatorname{coth}\left(\frac{\hbar W}{2 K_{B} T}\right)
\end{aligned}
$$

We note that minimizing $F_{v}$ with respect to variational parameters in $\mathrm{H}_{0}$ produces self-consistent equations for these parameters and the thermally averaged potential $\langle\mathrm{U}\rangle$ is reduced to

$$
\begin{aligned}
\langle\mathrm{U}\rangle=f^{\prime} & \frac{\sigma}{\alpha^{2}}+f \frac{\lambda}{\alpha^{2}} \\
& +\mathrm{V}_{0}\left\{\frac{\mathrm{A}^{2}}{2} \exp [2(\lambda+\sigma)] \cosh \left(2 \alpha\langle w\rangle_{T}\right)-2 \mathrm{Aexp}\left(\frac{\lambda+\sigma}{2}\right) \cosh \left(\alpha\langle w\rangle_{T}\right)\right\}
\end{aligned}
$$

Using equations (13), (15), (16), and (21) the resulting expression for the free energy is

$$
\mathrm{F}_{\mathrm{V}}=\mathrm{F}_{0}+\mathrm{N}\left\{\begin{array}{l}
f^{\prime} \frac{\sigma}{\alpha^{2}}+f \frac{\lambda}{\alpha^{2}}-\frac{\mathrm{m} \omega^{2} \lambda}{2 \alpha^{2}}-\frac{\mathrm{MW}^{2} \sigma}{2 \alpha^{2}} \\
+\mathrm{V}_{0}\left\{\frac{\mathrm{A}^{2}}{2} \exp [2(\lambda+\sigma)] \cosh \left(2 \alpha\langle w\rangle_{T}\right)-2 \operatorname{Aexp}\left(\frac{\lambda+\sigma}{2}\right) \cosh \left(\alpha\langle w\rangle_{T}\right)\right\}
\end{array}\right\}
$$

The trial parameters $\mathrm{W}$ and $\omega$ are variational parameters which may be determined by minimizing $\mathrm{F}_{\mathrm{v}}$ with respect to $\mathrm{W}$ and $\omega$. Minimizing expression (22) with respect to $\mathrm{W}$ and $\omega$, yields two equations which have to be solved self-consistently and we obtain 


$$
\frac{\mathrm{m} \omega^{2}}{2}=\alpha^{2}\left\{\frac{\mathrm{f}}{\alpha^{2}}+\mathrm{V}_{0}\left\{\begin{array}{l}
\mathrm{A}^{2} \exp (2(\lambda+\sigma)) \cosh \left(2 \alpha\langle w\rangle_{T}\right) \\
-\mathrm{A} \exp \left(\frac{\lambda+\sigma}{2}\right) \cosh \left(\alpha\langle w\rangle_{T}\right)
\end{array}\right\}\right\}
$$

and

$$
\frac{\mathrm{MW}^{2}}{2}=\alpha^{2}\left\{\frac{\mathrm{f}^{\prime}}{\alpha^{2}}+\mathrm{V}_{0}\left\{\begin{array}{l}
\mathrm{A}^{2} \exp (2(\lambda+\sigma)) \cosh \left(2 \alpha\langle w\rangle_{T}\right) \\
-\operatorname{Aexp}\left(\frac{\lambda+\sigma}{2}\right) \cosh \left(\alpha\langle w\rangle_{T}\right)
\end{array}\right\}\right\}
$$

Using equations (19), (20), (23), and (24), the frequencies $\mathrm{W}$ and $\omega$ are determined as functions of variables $\langle w\rangle_{T}$ and $\mathrm{T}$. Next by placing $\omega=\omega\left(\langle w\rangle_{T}, \mathrm{~T}\right)$ and $\mathrm{w}=\mathrm{w}\left(\langle w\rangle_{T}, \mathrm{~T}\right)$ into $\mathrm{F}_{\mathrm{v}}$, one can obtain a free energy which is represented as functions of $\langle w\rangle_{T}$ and $\mathrm{T}$, that is $\mathrm{F}_{\mathrm{v}}=\mathrm{F}_{\mathrm{v}}\left[\langle w\rangle_{T}, \mathrm{~T}\right)$.

By the use of this free energy, the thermal equilibrium relative displacement $\langle w\rangle_{T}$, at zero pressure, is determined by the expression

$$
\begin{array}{ll}
\frac{\partial \mathrm{F}_{\mathrm{V}}}{\partial\langle w\rangle_{T}}=0, & \text { for } \mathrm{A} \geq \exp \left[-\frac{3}{2}(\lambda+\sigma)\right] \\
\langle w\rangle_{T}= \begin{cases}0 & \text { for } \mathrm{A}<\exp \left[-\frac{3}{2}(\lambda+\sigma)\right] \\
\frac{1}{\alpha} \operatorname{arccosh}\left\{\exp \left[\ln \frac{1}{\mathrm{~A}}-\frac{3}{2}(\lambda+\sigma)\right]\right\} & \text {. }\end{cases}
\end{array}
$$

where the approximations for the hyperbolic cosines have been used.

It can be easily seen that Eq. (25) possesses two sets of solutions for the order parameter: $\langle w\rangle_{T}=0$ corresponds to the paraelectric phase (P.E phase), and $\langle w\rangle_{T} \neq 0$ corresponds to the ferroelectric phase (F.E phase).

\section{IV-Determination of the critical ferroelectric phase transition temperature.}

We now proceed to the calculation of the critical ferroelectric phase transition temperature in our two-component model. The critical temperature is determined by the condition that the system possesses the same free energy in both phases, that is

$$
\mathrm{F}\left(<w>_{T}>0, \mathrm{~T}_{\mathrm{c}}\right)=\mathrm{F}\left(<w>_{T}=0, \mathrm{~T}_{\mathrm{c}}\right) .
$$

It is also useful to have at hand a quantitative picture of how the transition temperature depends on the model parameters such as the ionic masses and the shell-core linear interaction potential parameters. 
At the transition temperature, $\langle w\rangle_{T}$ vanishes and then we have approximatively

$$
(\sigma+\lambda)_{T_{c}}=\frac{2}{3} \ln \left(\frac{1}{\mathrm{~A}}\right)
$$

Using equations (19) and (20), and considering that the variance at the critical point is found from (25) by letting $\langle w\rangle_{T} \rightarrow 0$ (Eq.(27)), we obtain a reduced critical temperature which must satisfy the following equation

$$
\begin{aligned}
\frac{4}{3 \alpha}\left(\frac{2 f f^{\prime}}{\mathrm{V}_{0}}\right)^{\frac{1}{2}} \ln \left(\frac{1}{\mathrm{~A}}\right)= & \hbar \alpha\left(\frac{f^{\prime}}{\mathrm{mV}_{0}}\right)^{\frac{1}{2}} \operatorname{coth}\left(\hbar\left(\frac{f}{2 \mathrm{~m}}\right)^{\frac{1}{2}} \frac{1}{\mathrm{k}_{\mathrm{B}} \mathrm{T}_{\mathrm{c}}}\right) \\
& +\hbar \alpha\left(\frac{f}{\mathrm{MV}_{0}}\right)^{\frac{1}{2}} \operatorname{coth}\left(\hbar\left(\frac{f^{\prime}}{2 \mathrm{M}}\right)^{\frac{1}{2}} \frac{1}{\mathrm{k}_{\mathrm{B}} \mathrm{T}_{\mathrm{c}}}\right)
\end{aligned}
$$

where $T_{c}$ is the phase-transition temperature.

We realize that this Eq.(28) doesn't permits us to derive an analytical expression for $T_{c}$ in the general case. Then we shall discuss the properties of these phases on the basis of Eq. (28) [or Eq.(27)] in two principal directions.

\section{IV.1 Case I: The shell-atom fluctuations are neglected.}

Hereafter, we neglect the shell- atom fluctuation $\lambda$ because shell atom is expected to be heavy compared with core atom and its fluctuation is expected to be small enough compared with the core fluctuation $\sigma$ and hardly exerts any much influence upon the critical phenomena of ferroelectric phase transition. Thus, developing (25), using $\lambda=0$ and then making use of the approximations for the hyperbolic cosines, we obtain

$$
<w>_{T}= \begin{cases}0 & \text { for } \sigma \geq \sigma_{\mathrm{c}} \\ \frac{1}{\alpha} \operatorname{arccosh}\left[\exp \left(\frac{3}{2}\left(\sigma_{\mathrm{c}}-\sigma\right)\right)\right] & \text { for } \sigma<\sigma_{\mathrm{c}}\end{cases}
$$

where

$$
\sigma_{\mathrm{c}}=\frac{2}{3} \ln \left(\frac{1}{\mathrm{~A}}\right)
$$

In order to develop the formula derived above, the following reduced parameters are introduced: 


$$
\begin{aligned}
& q=\left(\frac{\hbar^{2} \alpha^{2}}{M V_{0}}\right)^{\frac{1}{2}} \\
& \varpi^{2}=\frac{m \omega^{2}}{4 \alpha^{2} V_{0}} \\
& \Omega^{2}=\frac{M W^{2}}{4 \alpha^{2} V_{0}} \\
& t=\frac{k_{B} T}{V_{0}}
\end{aligned}
$$

The parameter $\mathrm{q}$, which represents quantum effect magnitude for the core atom, does not originate from the tunneling motion of the core atom but rather from quantum vibrations such as a zero-point oscillation.

By using these parameters, the core atom fluctuations $\sigma$ can be rewritten by

$$
\sigma=\frac{q}{4 \Omega} \operatorname{coth}\left(\frac{q \Omega}{t}\right)
$$

which can also give

$$
\mathrm{t}=\frac{\mathrm{q} \Omega}{\operatorname{arccoth}\left(\frac{4 \Omega \sigma}{\mathrm{q}}\right)}
$$

From Eqs.(23), (24) and (29), when $\lambda=0$ the relative displacement, the frequencies of shell and core atoms, respectively can be obtained as

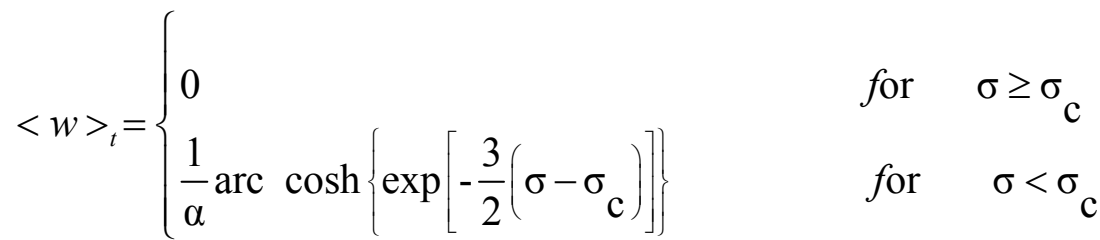

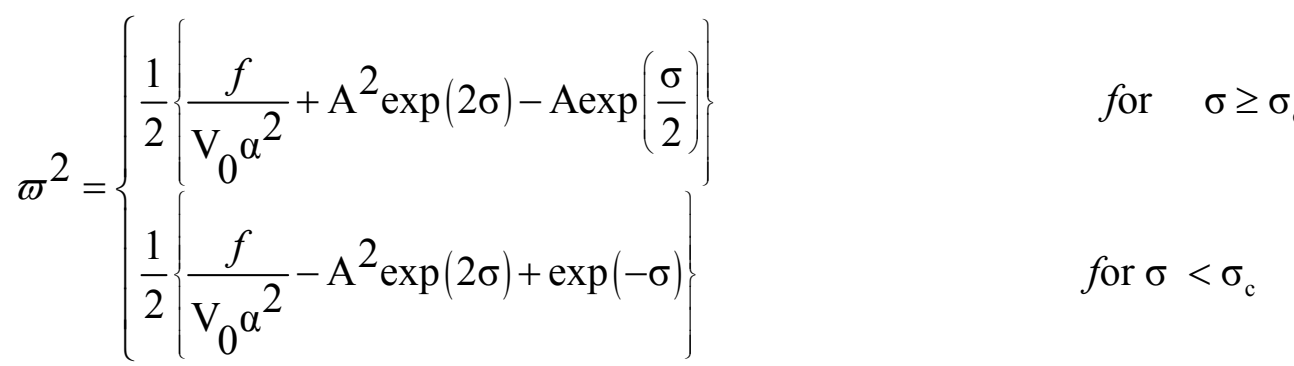




$$
\Omega^{2}= \begin{cases}\frac{1}{2}\left\{\frac{f^{\prime}}{\mathrm{V}_{0} \alpha^{2}}+\mathrm{A}^{2} \exp (2 \sigma)-\mathrm{A} \exp \left(\frac{\sigma}{2}\right)\right\} & \text { for } \sigma \geq \sigma_{\mathrm{c}} \\ \frac{1}{2}\left\{\frac{f^{\prime}}{\mathrm{V}_{0} \alpha^{2}}-\mathrm{A}^{2} \exp (2 \sigma)+\exp (-\sigma)\right\} & \text { for } \sigma<\sigma_{\mathrm{c}}\end{cases}
$$

Accordingly, the order parameter of this model is the thermal averages of the relative displacement $\langle w\rangle_{t}$. The case $\langle w\rangle_{t}=0$ corresponds also to an undisplaced solution whereas $\langle w\rangle_{t} \neq 0$ corresponds to a displaced one. The variable $t$ comes from Eq.(31).

Since the statistic properties of the system are not significantly affected, we have from Eq.(30) and (20), the approximate reduced phase transition temperature given by

$$
t_{\mathrm{c}}=\frac{\mathrm{q}}{\alpha}\left(\frac{f^{\prime}}{2 \mathrm{~V}_{0}}\right)^{\frac{1}{2}} \frac{1}{\operatorname{arccoth}\left(\frac{4}{3} \frac{1}{\mathrm{q} \alpha}\left(\frac{2 f^{\prime}}{\mathrm{V}_{0}}\right)^{\frac{1}{2}} \ln \left(\frac{1}{\mathrm{~A}}\right)\right)} .
$$

Using the relationship between the inverse hyperbolic functions and the logarithm, this expression of the reduced phase transition temperature may be expressed as

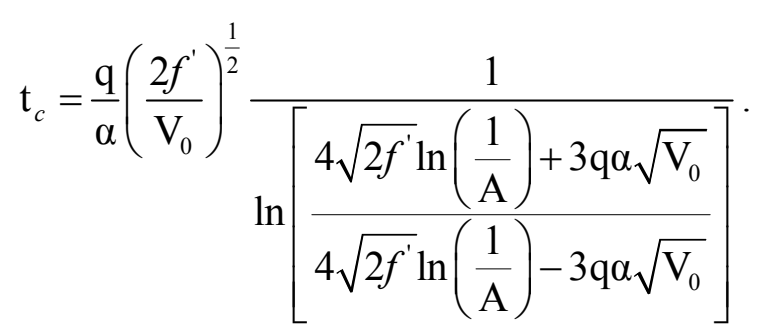

It is also interesting to note that the expression for $t_{c}$ in the classical limit can be worked out exactly for our model restricting to the simplified assumption made in Eq.(29).

The displacement type phase transition appears in the weak anharmonicity limit. In this case, the height of the potential barrier in the double-well potential (2), $\Delta \mathrm{V}=\mathrm{V}_{0}(1-\mathrm{A})^{2}$ is small as compared with the energy of intercell interactions between nearest-neighbors $\frac{f f^{\prime} w_{0}^{2}}{f+f^{\prime}}>>\Delta \mathrm{V}$, where $w_{0}$ locates the potential minima (case of strong coupling between nearest-neighbors relative to the energy barrier of the double-well potential.) $[30,31]$. This corresponds to the displacive regime where the displacement pattern varies smoothly over lattice spacing.

Under some constraints of the model parameters (high-temperature approximation or small quantum effect), an analytical expression for $t_{c}$ can be estimated as

$$
\mathrm{t}_{c}=\frac{2}{3}\left(\frac{2 f^{\prime}}{\mathrm{V}_{0} \alpha^{2}}\right) \ln \left(\frac{1}{\mathrm{~A}}\right)
$$

This reduced phase transition temperature has the same form as the one obtained by Konwent on the application of a double-well potential in the theory of ferroelectric phase transition [15]. On the other 
hand, the order-disorder type phase transition can takes place in the limit of strong anharmonicity when the potential barrier between two minima of the potential (2) is much greater than the coupling between nearest-neighbors $\Delta \mathrm{V}=\mathrm{V}_{0}\left(1-\mathrm{A}^{2}\right)>>\frac{f f^{\prime} \mathrm{w}_{0}^{2}}{f+f^{\prime}}$.

\section{IV.2 Case II: High-temperature regime}

In the high-temperature, that is for large $\mathrm{T}\left(2 \mathrm{k}_{\mathrm{B}} \mathrm{T}>\hbar \omega\right.$ and $\left.2 \mathrm{k}_{\mathrm{B}} \mathrm{T}>\hbar \mathrm{W}\right)$, the hyperbolic cotangent can be replaced by the reciprocal of its argument. An analytical expression for $T_{c}$ can then be derived from Eq. (28) which gives,

$$
\mathrm{k}_{B} \mathrm{~T}_{c}=\frac{4}{3} \frac{f_{D}}{\alpha^{2}} \ln \left(\frac{1}{\mathrm{~A}}\right)
$$

where

$$
f_{D}=\frac{f f^{\prime}}{f+f^{\prime}}
$$

This expression of transition temperature is mass independent. Obviously, the magnitude of $T_{c}$ is mainly determined by the intermolecular shell-shell coupling $\mathrm{f}$ and core-core coupling f' and the deformability parameter A which is intimately related to the shell ion polarizability.

A variation in $T_{c}$ was achieved by changing the depth of the double-well potential given by A (see Fig.8).

\section{V-Special case}

The solutions obtained in the last section are not valid in the case where $\mathrm{M}=\mathrm{m}, f=f^{\prime}$. The Hamiltonian of the model is reduced to the simplified one and we can obtain a more convenient form by introducing new variables that would correspond to normal coordinates in the linear limit. The canonical transformation to new variables $x_{i}=\left(u_{i}+v_{i}\right) / \sqrt{2} ; y_{i}=\left(u_{i}-v_{i}\right) / \sqrt{2}$ is revealing in this special case. Under this transformation, the Hamiltonian becomes

$$
\begin{aligned}
\mathrm{H}=\sum_{i}\left[\frac{1}{2} \mathrm{~m} \dot{x}_{i}^{2}+\right. & \left.\frac{1}{2} \mathrm{~m} \dot{y}_{i}^{2}+\frac{1}{2} f\left(x_{i+1}-x_{i}\right)^{2}+\frac{1}{2} f\left(y_{i+1}-y_{i}\right)^{2}+\mathrm{V}\left(\sqrt{2} y_{i}\right)\right] \\
=\sum_{i}\left[\frac{1}{2} \mathrm{~m} \dot{x}_{i}^{2}\right. & \left.+\frac{1}{2} f\left(x_{i+1}-x_{i}\right)^{2}\right] \\
& +\sum_{i}\left[\frac{1}{2} \mathrm{~m} \dot{y}_{i}^{2}+\frac{1}{2} f\left(y_{i+1}-y_{i}\right)^{2}+\mathrm{V}\left(\sqrt{2} y_{i}\right)\right]
\end{aligned}
$$


The part of the Hamiltonian that depends on the variable $x_{i}$ is decoupled from the $y_{i}$ part and corresponds merely to a harmonic chain without substrate potential. Therefore, we henceforth consider only the part of $\mathrm{H}$, which depends on $y_{i}[32,33]$,

$$
\mathrm{H}_{y}=\sum_{i}\left[\frac{1}{2} \mathrm{~m} \dot{y}_{i}^{2}+\frac{1}{2} f\left(y_{i+1}-y_{i}\right)^{2}+\mathrm{V}_{0}\left\{\begin{array}{l}
\frac{\mathrm{A}^{2}}{4}\left[\exp \left(2 \sqrt{2} \alpha y_{i}\right)+\exp \left(-2 \sqrt{2} \alpha y_{i}\right)\right] \\
-\mathrm{A}\left[\exp \left(\sqrt{2} \alpha y_{i}\right)+\exp \left(-\sqrt{2} \alpha y_{i}\right)\right]
\end{array}\right\}\right]
$$

and describes the dynamics of the ferroelectric phase transition we are interested in. Introducing the SCEM, we have a trial Hamiltonian given by

$$
\mathrm{H}_{0}=\frac{1}{2} \sum_{i=1}^{\mathrm{N}} \mathrm{m} \dot{y}_{i}^{2}+\frac{\mathrm{k}}{2} \sum_{i=1}^{\mathrm{N}}\left(\delta y_{i}\right)^{2}
$$

The free energy is then

$$
\mathrm{F}_{\mathrm{v}}=\mathrm{Nk}_{B} \operatorname{Tln}\left(2 \sinh \left(\frac{\hbar \omega}{\mathrm{k}_{B} \mathrm{~T}}\right)\right)+\mathrm{N}\left\{f \frac{\lambda}{\alpha^{2}}-\frac{\mathrm{m} \omega^{2} \lambda}{2 \alpha^{2}}+\mathrm{V}_{0}\left\{\begin{array}{l}
\frac{\mathrm{A}^{2}}{2} \exp (4 \lambda) \cosh \left(2 \sqrt{2} \alpha\langle y\rangle_{T}\right) \\
-2 \operatorname{Aexp}(\lambda) \cosh \left(\sqrt{2} \alpha\langle y\rangle_{T}\right)
\end{array}\right\}\right\}
$$

where $\lambda$ and $\omega$ are given by Eq.(14) and (19).

Using the same approach and considering $\omega$ and $\langle y\rangle_{T}$ as variational parameters [34, 35], the condition for free energy to be stationary are then equivalent to the following self-consistent equations

$$
\begin{aligned}
& \frac{\mathrm{m} \omega^{2}}{2}=\alpha^{2}\left\{\frac{f}{\alpha^{2}}+\mathrm{V}_{0}\left\{\begin{array}{ll}
2 \mathrm{~A}^{2} \exp (4 \lambda) \cosh \left(2 \sqrt{2} \alpha\langle y\rangle_{T}\right) \\
-2 \operatorname{Aexp}(\lambda) \cosh \left(\alpha \sqrt{2}\langle y\rangle_{T}\right)
\end{array}\right\}\right\} \\
& <y>_{T}= \begin{cases}0 & \text { for } \lambda \geq \lambda \\
\frac{1}{\sqrt{2} \alpha} \operatorname{arccosh}\left\{\exp \left[-3\left(\lambda-\lambda_{\mathrm{c}}\right)\right]\right\} & \text { for } \lambda<\lambda_{\mathrm{c}}\end{cases}
\end{aligned}
$$

where

$$
\lambda_{c}=\frac{1}{3} \ln \left(\frac{1}{A}\right)
$$

By Eqs.(46) and (47), the frequency $\omega$ are determined as functions of variable $\langle y\rangle$ and $\mathrm{T}$.

Thus 


$$
\varpi^{2}= \begin{cases}\frac{1}{2}\left(\frac{f}{\mathrm{~V}_{0} \alpha^{2}}\right)+\mathrm{A}^{2} \exp (4 \lambda)-\mathrm{A} \exp (\lambda) & \text { for } \lambda \geq \lambda_{\mathrm{c}} \\ \frac{1}{2}\left(\frac{f}{\mathrm{~V}_{0} \alpha^{2}}\right)-\mathrm{A}^{2} \exp (4 \lambda)+\exp (-\lambda) & \text { for } \lambda<\lambda_{\mathrm{c}}\end{cases}
$$

where

$$
\varpi^{2}=\frac{m \omega^{2}}{4 \alpha^{2} V_{0}} .
$$

In this case, the expression of critical transition temperature is given by

$$
\mathrm{t}_{\mathrm{c}}=\frac{\mathrm{q}}{\alpha}\left(\frac{f}{2 \mathrm{~V}_{0}}\right)^{\frac{1}{2}} \frac{1}{\operatorname{arccoth}\left(\frac{2 \sqrt{2}}{3 \mathrm{q} \alpha}\left(\frac{f}{\mathrm{~V}_{0}}\right)^{\frac{1}{2}} \ln \left(\frac{1}{\mathrm{~A}}\right)\right)}
$$

The high temperature approximation of analytical expression of the reduced ferroelectric phase transition temperature is then

$$
\mathrm{t}_{c}=\frac{2}{3} \frac{f}{\mathrm{~V}_{0} \alpha^{2}} \ln \left(\frac{1}{\mathrm{~A}}\right)
$$

It is important to note that the above expression (51) of the reduced critical transition temperature is equal to the expression (39) divided by two.

Obviously, in this case, the mass independence of the critical phase transition temperature is evident. This theoretical reduced ferroelectric transition temperature $t_{c}$ determines the boundary of the region where a non zero value of order parameter becomes stable.

\section{VI- Application to $\mathrm{K}_{2} \mathrm{SeO}_{4}$ crystal}

The conventional model presented in previous sections, based on the anisotropic non-linear polarizability of the oxygen ion and its homologues whose dynamics has been taken into account in terms of a local symmetric double Morse-type potential, can be applied in the ferroelectric phase of $\mathrm{K}_{2} \mathrm{SeO}_{4}$. Potassium selenate is the best-known materials of the $\mathrm{A}_{2} \mathrm{BX}_{4}$ family having an orthorhombic space group Pna2 ${ }_{1}$. The symbol A represents an alkali-metal ion or equivalent monovalent complex such as $\mathrm{NH}_{4}{ }^{+}$or $\mathrm{N}_{(}\left(\mathrm{CH}_{3}\right)_{4}{ }^{+}$. The symbol $\mathrm{BX}_{4}$ represents a divalent tetrahedral complex such as $\mathrm{SeO}_{4}{ }^{2-}$, $\mathrm{ZnCl}_{4}{ }^{2-}$ or $\mathrm{ZnBr}_{4}{ }^{2-}$. At low temperatures, different but related structure-modulated phases are found along the crystal family [36-41].

Potassium selenate was found to undergo two successive phase transformations at $T_{1}=129.5 \mathrm{~K}$ and $\mathrm{T}_{\mathrm{c}}$ $=93 \mathrm{~K}$, the latter being a ferroelectric phase transformation with spontaneous polarization along the $\mathrm{c}$ axis $[40,42]$. The lock-in transition at $93 \mathrm{~K}$ is characterized by a tripling of the unit cell. As pointed 
out by Cochran [43], and according to the notation of Kalman et al. [38], the two symmetrically nonequivalent potassium ions $\mathrm{K}_{\alpha}$ and $\mathrm{K}_{\beta}$ and the $\mathrm{SeO}_{4}$ complex are taken to be equivalent. A reduction of the $\mathrm{K}_{2} \mathrm{SeO}_{4}$ unit cell to a CsCl-type structure is possible by assuming that the $\mathrm{K}_{\beta}$ ions are negligible, while the $\mathrm{K}_{\alpha}$ ions have twice the mass of the potassium ion. By means of this simplification it is possible to apply the pseudo-one dimensional models to $\mathrm{K}_{2} \mathrm{SeO}_{4}[44,45]$. The lattice constant a taken in the polarisation direction has been also determined from the experimental inelastic neutronscattering data [39].

The choice of the value of parameter $f^{\prime}$ 'were motivated by the fact that the lock-in phase transition for the potassium selenate which is first order phase transition can only take place when $f$ ' is small [46].

The mass $\mathrm{M}$ is given by twice the potassium ion mass while the complex $\mathrm{SeO} 4$ gives $\mathrm{m}$. We then have the following set of parameters for the $\mathrm{K}_{2} \mathrm{SeO}_{4}$ at the phase transition point $[14,47,48]$ :

$\mathrm{M}=1.298 \times 10^{-22} \mathrm{~g}$

$\mathrm{m}=2.373 \times 10^{-22} \mathrm{~g}$

$f=1.183 \times 10^{+4} \mathrm{gs}^{-2}$;

$f^{\prime}=1.75 \times 10^{+2} \mathrm{gs}^{-2}$;

$\alpha=2.89 \AA^{-1}$;

$\mathrm{V}_{0}=0.03 \mathrm{eV}$;

$\mathrm{r}_{0}=0.98 \AA$;

$\mathrm{N}=100$.

Moreover, numerical studies of the critical phase transition temperature are performed at the different values of $A$ and therefore, we get by this procedure in the case $\mathrm{I}$, for $\mathrm{A}=0.01, \mathrm{~T}_{\mathrm{c}} \approx 92.5 \mathrm{~K}$, while for $\mathrm{A}$ $=0.0187, \mathrm{~T}_{\mathrm{c}} \approx 80 \mathrm{~K}$. In the case II, for $\mathrm{A}=0.01, \mathrm{~T}_{\mathrm{c}} \approx 91.5 \mathrm{~K}$, while for $\mathrm{A}=0.0187, \mathrm{~T}_{\mathrm{c}} \approx 79 \mathrm{~K}$. The experimental temperature of the lock-in phase transition at zero pressure is $93 \mathrm{~K}$ [49]. This value of critical phase transition temperature is small than the experimental value but approach it.

In Figs. 2 are shown the representations of the free energy versus the reduced temperature for different values of the relative displacement $\langle w\rangle_{T}$. In each case, we realize that the free energy decreases as the temperature increases. Fig.2a characterizes the ferroelectric phase whereas Fig.2b refers to the incommensurate-paraelectric regime.

A plot of $t_{c}$ as a function of the parameter $A$ is shown in Fig.3. We observe that, $t_{c}$ decreases very rapidly to zero as A increases. In other words, the influence of the deformability parameter on the critical phase transition temperature is important. Fig. $3 \mathrm{~b}$ which shows the dependence of the reduced transition temperature $t_{c}$ on the model parameter $\mathrm{R}$ allows us to study it influence on the properties of the ferroelectric crystals. Then, we have notified that phase transition to the ordered phase is possible only if the parameter $\mathrm{R}$ is greater than some critical value $\mathrm{R}_{\mathrm{c}}$.

In Fig.4, the curves of the order parameter $\langle w\rangle_{T}$ against the core fluctuation $\sigma$ are plotted for three values of the parameter $\mathrm{A}$, namely $\mathrm{A}=0.010, \mathrm{~A}=0.0187$, and $\mathrm{A}=0.050$. When $\sigma<\sigma_{\mathrm{c}}$, the order 
parameter is different from zero and decreases slowly to zero as the core fluctuation $\sigma$ increases. This means that the mean relative displacement is different from zero and the shell-atom displacement is also different and greater than the core-atom displacement. Here is the main characteristic of the displaced solution, which corresponds to the ferroelectric phase.

Contrastingly, when $\sigma \geq \sigma_{\mathrm{c}}$, the order parameter is always equal to zero regardless of the core fluctuation. In this case, the shell-atom displacement is always equal to the core-atom displacement. Such a situation characterizes the symmetric disposition of core and shell atoms in the model, which attends to the undisplaced solution.

In Figs.5, the curves of shell frequencies $\omega$ and core frequencies $\Omega$ for different values of the deformability parameter A are plotted against the core fluctuation $\sigma$. When the value of A is small and $\sigma$ is also small $\left(\sigma<\sigma_{c}\right)$, the core and shell atoms vibrate locally about one of the two equilibrium sites (see for example Fig.5a and Fig.5b). These vibrations correspond approximatively to the core-shell bond-stretching vibrations softens as $\sigma$ increases, and it becomes too small at $\sigma=\sigma_{\mathrm{c}}$ for the core frequency case. The softening of the vibration near $\sigma=\sigma_{\mathrm{c}}$ is based on the instability of asymmetric stretching vibrations of core-shell bond. When $\sigma$ is large $\left(\sigma>\sigma_{c}\right)$, the shell and core atoms oscillate from one equilibrium site to the other thereby making the averaged position of shell atom at the center core-shell bond.

On the other hand, we have represented the relationship between the core fluctuation $\sigma$ and the temperature $\mathrm{t}$ (see Fig.6). It appears for the evolution of $\sigma$, that it increases continuously from 0 to $\sigma_{\mathrm{c}}$ according as $t$ increases from zero to $t_{1}$ and decreases from $t_{1}$ to $t_{c}$. Furthermore at $t_{c}$, an abrupt transition from an asymmetric bond to a symmetric bond appears. This is characterized by a rapid change of direction for the curve (inflexion point). At this point, we can strongly consider the zeropoint fluctuation value $\sigma_{0}$ because it exhibits the geometric isotope effect most remarkably. This zeropoint fluctuation is caused by the zero-point oscillation of shell-atom. This isotope effect is not our main problem here and must be treated in the future. For $\mathrm{t}>\mathrm{t}_{\mathrm{c}}$, the core fluctuation $\sigma$ increases asymptotically and presents the saturation value for large reduced temperature; that is the temperature independence of the core fluctuation is evident.

In Fig.7 is plotted the variation of the temperature with the order parameter value $\langle\mathrm{w}\rangle_{T}$ for different values of A. This figure shows that order parameter decreases as the temperature increases. For each value of $\mathrm{A}$, the system has three sets of solutions. When $\mathrm{t}\left\langle\mathrm{t}_{\mathrm{c}}\right.$, there is only one solution with $\langle\mathrm{w}\rangle_{T} \neq$ 0 corresponding to the ferroelectric phase. When $t_{c} \leq t \leq t_{1}$, the equation has three solutions: one with $\langle w\rangle_{T}=0$ and two others with $\langle w\rangle_{T} \neq 0$. 
When $\mathrm{t}>\mathrm{t}_{1}$ there is also one possible solution with $\langle\mathrm{w}\rangle_{T}=0$. As it is clear, when we analyze the behavior of the free energy near the reduced critical temperature $\mathrm{t}_{\mathrm{c}}$, the solution with $\langle\mathrm{w}\rangle_{T} \neq 0$ is unstable for $t \geq t_{c}$, and only $\langle\mathrm{w}\rangle_{T}=0$ is a stable solution. This means that the system changes discontinuously by a jump of the order parameter from the ferroelectric phase to the incommensurateparaelectric phase when the reduced temperature increase from below $t_{c}$ to above. Thus, the system exhibits a first-order phase transition. It is also observed from Fig.7 that $t_{1}$ is the limiting reduced temperature below which the system can be found in the ferroelectic phase. Again from the analysis of the free energy, the ferroelectric phase is metastable but the paraelectric phase is stable in the neighborhood of $t_{1}$. Hence, the system remains in the paraelectric phase when the temperature drops down to $t_{1}$. On the other hand, the paraelectric phase is metastable and the ferroelectric phase is stable at $t_{c}$ and the system remain in the ferroelectric phase as the temperature increases to $t_{c}$. This implies that $t_{c}$ is the limiting temperature for the stable paraelectric phase. Therefore paraelectric and ferroelectric phases coexist when the temperature is within the interval $t_{c}<t<t_{1}$. We then conclude that within this interval, there exists a temperature at which the stability of the ferroelectric and paraelectric phases are equals. This is the Curie temperature of the first-order phase transition [50].

In Fig. 8 is plotted the variation of the phase transition temperature $T_{c}$ vs the deformability parameter $\mathrm{A}$. We also observe that, $\mathrm{T}_{\mathrm{c}}$ decreases very rapidly to zero as $\mathrm{A}$ increases.

\section{VII- Conclusion}

In this paper, we have calculated the critical ferroelectric phase transition temperature of the model based on the local nonlinear chalcogenide ion polarizability. The calculation, which is done on the basis of the Self-Consistent Einstein Model, presupposes that we neglect the shell-atom fluctuation compared with the core-atom fluctuation on the one hand, and in the high-temperature regime, on the other hand. The method was illustrated to $\mathrm{K}_{2} \mathrm{SeO}_{4}$. Good agreement is found for the known experimental critical temperature when $\mathrm{A}=0.010$ for both cases. Of course, this can be applied to other problems of physics of similar nature.

Acknowledgments: One of the authors (C.M.N.) appreciates the facilities provided by the Abdus Salam International Centre for Theoretical Physics, Trieste, Italy for the improvement of this work. She acknowledges the Swedish International Development Cooperation Agency (SIDA) for financial support. This work was done within the framework of the Associateship Scheme of the Abdus Salam ICTP. 


\section{APPENDIX}

In this appendix we present only the main results useful to obtain the expression of the main value of the potential $\langle\mathrm{U}\rangle$. We consider a thermal average of the generalized potential $\mathrm{V}_{\mathrm{G}}(\mathrm{R}+\mathrm{u})$ with respect to the harmonic Hamiltonian where $\mathrm{R}$ is the thermal equilibrium relative displacement and $\mathrm{u}$, the fluctuation about this displacement using Fourier components

$$
\mathrm{V}_{\mathrm{G}}(\mathrm{r})=\int_{-\infty}^{+\infty} \tilde{\mathrm{V}}_{\mathrm{G}}(\mathrm{q}) \mathrm{e}^{\mathrm{iqr}} \mathrm{dq}
$$

and

$$
\mathrm{V}_{\mathrm{G}}(\mathrm{q})=\frac{1}{2 \pi} \int_{-\infty}^{+\infty} \mathrm{V}_{\mathrm{G}}(\mathrm{r}) \mathrm{e}^{-\mathrm{iqr}} \mathrm{dr}
$$

By the use of equation (A1), the thermal average potential $<\mathrm{V}_{\mathrm{G}}(\mathrm{R}+\mathrm{u})>$ can be expressed as

$$
<\mathrm{V}_{\mathrm{G}}(\mathrm{R}+\mathrm{u})>=\int_{-\infty}^{+\infty} \widetilde{V}_{G}(q) e^{i q R}<e^{i q u}>d q
$$

Since $\langle\cdots\rangle$ [defined by Eq. (11)] is the thermal average with respect to the harmonic Hamiltonian and using the following results

$$
\begin{aligned}
& <\mathrm{u}_{\mathrm{k}}^{2 \mathrm{n}+1}>=0, \\
& \text { and } \\
& <\mathrm{u}_{\mathrm{k}}^{2 \mathrm{n}}>=\frac{(2 \mathrm{n}) !}{2^{\mathrm{n}} \mathrm{n} !}<\mathrm{u}^{2}>^{\mathrm{n}},
\end{aligned}
$$

one can obtain

$$
<\mathrm{e}^{\mathrm{iqu}}>=\exp \left[\frac{-\mathrm{q}^{2}}{2}<\mathrm{u}^{2}>\right]
$$

Then using (A2), (A1) and (A3) yields

$$
<\mathrm{V}_{\mathrm{G}}(\mathrm{R}+\mathrm{u})=\frac{1}{2 \pi} \int_{-\infty}^{+\infty} \mathrm{V}_{\mathrm{G}}(\mathrm{r})\left[\int_{-\infty}^{+\infty} \mathrm{e}^{\mathrm{iq}(\mathrm{R}-\mathrm{r})} \exp \left(\frac{-<\mathrm{u}^{2}>\mathrm{q}^{2}}{2}\right) \mathrm{dq}\right] \mathrm{dr}
$$

where the integral in the large square bracket $[\ldots]$ becomes:

$$
\begin{aligned}
\int_{-\infty}^{+\infty} \mathrm{e}^{\mathrm{iq}(\mathrm{R}-\mathrm{r})} \exp \left(\frac{-<\mathrm{u}^{2}>\mathrm{q}^{2}}{2}\right) \mathrm{dq} & =\int_{-\infty}^{+\infty} \mathrm{e}^{-\frac{<\mathrm{u}^{2}>}{\mathrm{q}}}\left[\left(\mathrm{q}-\frac{\mathrm{i}(\mathrm{R}+\mathrm{r})}{<\mathrm{u}^{2}>}\right)^{2}-\left(\frac{\mathrm{i}(\mathrm{R}-\mathrm{r})}{<\mathrm{u}^{2}>}\right)^{2}\right] \\
& =\mathrm{e}^{-\frac{(\mathrm{R}-\mathrm{r})^{2}}{2<\mathrm{u}^{2}>} \int_{-\infty}^{+\infty}} \mathrm{e}^{-\frac{<\mathrm{u}^{2}>}{2}}\left(\mathrm{q}-\frac{\mathrm{i}(\mathrm{R}-\mathrm{r})}{<\mathrm{u}^{2}>}\right) \mathrm{dq}
\end{aligned}
$$

Using the result $\int_{0}^{+\infty} \mathrm{e}^{-\mathrm{ax}^{2}} \mathrm{dx}=\frac{1}{2} \sqrt{\frac{\pi}{\mathrm{a}}}$ and after some manipulations the following result can be written 


$$
\int_{-\infty}^{+\infty} \mathrm{e}^{\mathrm{iq}(\mathrm{R}-\mathrm{r})} \exp \left[\frac{-<\mathrm{u}^{2}>\mathrm{q}^{2}}{2}\right] \mathrm{dq}=\left[\frac{2 \pi}{<\mathrm{u}^{2}>}\right]^{\frac{1}{2}} \exp \left[\frac{-(\mathrm{R}-\mathrm{r})^{2}}{2<\mathrm{u}^{2}>}\right] .
$$

By using Eq. (A7), we finally obtain thermal averaged potential expressed as

$$
<\mathrm{V}_{\mathrm{G}}(\mathrm{R}+\mathrm{u})>=\frac{1}{\left[2 \pi<\mathrm{u}^{2}>\right]^{\frac{1}{2}}} \int_{-\infty}^{+\infty} \mathrm{V}_{\mathrm{G}}(\mathrm{r}) \exp \left[\frac{-(\mathrm{R}-\mathrm{r})^{2}}{2<\mathrm{u}^{2}>}\right] \mathrm{dr}
$$

This expression allows us to calculate the thermal average of the total potential energy $<\mathrm{U}>$ using expression (5b). This yields the equation:

$$
\langle\mathrm{U}\rangle=\sum_{j=1}^{N}\left[\left\langle f\left(\delta u_{\mathrm{j}+1}-\delta u_{\mathrm{j}}\right)^{2}\right\rangle+\left\langle f^{\prime}\left(\delta v_{j+1}-\delta v_{j}\right)^{2}\right\rangle+\left\langle\mathrm{V}\left(\left\langle w_{j}\right\rangle_{T}+\delta v_{\mathrm{j}}-\delta u_{\mathrm{j}}\right)\right\rangle\right]
$$

where

$$
\left\langle w_{j}\right\rangle_{\mathrm{T}}=\left\langle v_{j}-\mathrm{u}_{\mathrm{j}}\right\rangle_{T} \approx\left\langle v_{j}\right\rangle_{T}-\left\langle u_{j}\right\rangle_{T}
$$

The thermal averages of the right hand side of Eq. (A9) can be concretely calculated by the application of the double Morse-type potential defined by Eq. (2) to $\mathrm{V}_{\mathrm{G}}(\mathrm{r})$ in Eq. (A8). This application yields in the following final result:

$$
\langle V(R+u)\rangle=V_{0}\left\{\frac{A^{2}}{2} \exp \left[2 \alpha^{2}\left\langle u^{2}\right\rangle\right] \cosh (2 \alpha R)-2 A \exp \left[\frac{\alpha^{2}}{2}\left\langle u^{2}\right\rangle\right] \cosh (\alpha R)\right\}
$$

and we can then obtain the mean value of the interaction potential

$$
\left\langle\mathrm{V}\left(\left\langle\mathrm{w}_{\mathrm{j}}\right\rangle_{T}+\delta v_{j}-\delta u_{j}\right)\right\rangle=2 \mathrm{~V}_{0}\left\{\begin{array}{l}
\frac{A^{2}}{4} \exp \left[2 \alpha^{2}\left(\left\langle\left(\delta v_{j}\right)^{2}\right\rangle+\left\langle\left(\delta u_{j}\right)^{2}\right\rangle\right)\right] \cosh \left(2 \alpha\left\langle w_{j}\right\rangle_{T}\right) \\
-A \exp \left(\frac{\alpha^{2}}{2}\left(\left\langle\left(\delta v_{j}\right)^{2}\right\rangle+\left\langle\left(\delta u_{j}\right)^{2}\right\rangle\right)\right) \cosh \left(\alpha\left\langle w_{j}\right\rangle_{T}\right)
\end{array}\right\}
$$

Also we have

$$
\begin{aligned}
& \left\langle f\left(\delta u_{j+1}-\delta u_{j}\right)^{2}\right\rangle=f\left(\left\langle\left(\delta u_{j+1}\right)^{2}\right\rangle+\left\langle\left(\delta u_{j}\right)^{2}\right\rangle\right) \\
& \left.\left.\left\langle f^{\prime}\left(\delta v_{j+1}-\delta v_{j}\right)^{2}\right\rangle=f^{\prime}\left(\left\langle\left(\delta v_{j+1}\right)^{2}\right\rangle+\right\rangle\left(\delta v_{j}\right)^{2}\right\rangle\right)
\end{aligned}
$$

All these expressions allow us to calculate and to compute the value of the free energy (22). 


\section{REFERENCES}

1- R.A. Cowley, Phys. Rev. Lett. 9, 159 (1962).

2- W. Cochran, Adv. Phys. 18, 157 (1969).

3- L. D. Landau, JETP.7, 19 (1937).

4- N. S. Gillis and T.R. Koehler, Phys. Rev. B4, 3971 (1971).

5- N. S. Gillis and T.R. Koehler, Phys. Rev. B5, 1925 (1972).

6- M. E. Lines, Phys. Rev.177, 797 (1968).

7- M. E. Lines, Phys. Rev. 177, 812 (1969).

8- E. Pytte and J. Feder, Phys. Rev. 187, 1077 (1969).

9- Y. Onodera, Prog. Theor. Phys. 44, 1477 (1970).

10- Y. Onodera, Prog. Theor. Phys. 45, 986 (1971).

11 - E. Pytte, Phys. Rev. B5, 3758 (1972).

12- G. Shirane, J. D. Axe, J. Harada, and J.P. Remeika, Phys. Rev. B2, 155 (1970).

13- H. Bilz, G. Benedek, and A. Bussmann-Holder, Phys. Rev. B35, 4840(1987).

14- C. M. Ngabireng and T. C. Kofané, Physica Scripta 55, 257 (1997).

15- H. Konwent, phys. stat. Sol. (b) 138, K7 (1986).

16- M. C. Lawrence and G. N. Robertson, Ferroelectrics 34, 179 (1981).

17- E. Matsushita and T. Matsubara, Progr. Theor. Phys. 67, 1 (1982).

18- S. Tanaka, , Phys. Rev.B42, 10488 (1990).

19- X. Duan and S. Scheiner, International Journal of Quantum chemistry: Quantum Biology Symposium 19 , (1992)109.

20- X. Duan and S. Scheiner, J. Mol. Struct. 270, 173 (1992).

21- A. V. Zolotaryuk, St. Pnevmatikos, A. V. Savin, Physica D51, 407 (1991).

22- A. V. Savin and A. V. Zolotaryuk, Phys. Rev. A44, 8167 (1991).

23- G. Kalosakas, A. V. Zolotaryuk, G.P. Tsironis and E.N. Economou, Phys. Rev. E56, 1088 (1997).

24- M. Peyrard (Ed.). “Nonlinear Excitations in Biomolecules”, Springer, Berlin, 1995.

25- N. S. Gillis, Phys. Rev. B11, 309 (1975).

26- N. S. Gillis and T.R. Koehler Phys. Rev. B9, 3806 (1974).

27- N F. Nabarro, “ Theory of Crystal Dislocations "(Clarendo, Oxford, 1967).

28- J. R. Morris and R. J. Gooding, Phys. Rev. B43, 6057 (1991).

29- R. P. Feynman, "Statistical Mechanics" (Benjamin-Cummings Reading, Massachusetts, 1972).

30- J. A. Krumshanl and J. R. Schrieffer, Phys. Rev. B11, 3535 (1975).

31 - A. O. Dobry, R.L. Migoni, and H. A. Ceccato, Phys. Rev. B38, 2801 (1988).

32- T. Dauxois and M. Peyrard, Phys. Rev. E47, 684 (1993 ).

33- T. Dauxois and M. Peyrard, Phys. Rev. E51, 4027 (1995).

34- T.R. Koehler and S. Gillis Phys. Rev. B13, 4183 (1976).

35- R. Blinc, B. Zeks, J. F. Sampaio, A. S. T. Pires, and F. C. Barreto, Phys. Rev. B20, 1991 (1979). 
36- K. Inoue, K. Suzuki, A. Sawada, Y. Ishibashi and Y. Takagi, J. Phys. Soc. Jpn 46, 608 (1979).

37- G. Gattow, Acta-Cryst. B15, 419 (1962).

38- A. Kalman, J. S. Stephens, and D. W. Gruickshank, Acta-Cryst. B26, 1451 (1970).

39- M. Lizumi, J. D. Axe, G. Shirane, and R. Shimaoka, Phys. Rev. B15, 4392 (1977).

40- K. Aiki, K. Hukaada and O. Matamura, J. Phys. Soc. Jpn 26, 1064 (1969).

41- H. Z. Cummins, Phys. Rep. 185, 2111(1990) and references therein.

42- K. Aiki and K. Hukaada, J. Phys. Soc. Jpn 26, 1066(1969).

43- W. Cochran, Adv. Phys.9, 387(1960).

44- A. Bussmann, H. Bilz and G. Benedek, Phys. Rev. B39, 9214 (1989).

45- A. Bussmann- Holder and H. Büttner, Phys. Rev. B41, 9581 (1990).

46- A. Bilz, H. Büttner, A. Bussman-Holder, W. Kress and U. Schröder, Phys. Rev. Lett. 48, 1982.

47- I. Etxebarria, R. M. Lynden-Bell, and J. M. Perez-Mato, Phys. Rev. B46, 13687 (1992).

48- H. Sugimoto and S. Ikeda, Phys. Rev. Lett. 67, 1306 (1991).

49- P. Echegut, F. Gervais and N. E. Massa, Phys. Rev. B34, 278 (1986).

50- C. L Wang, Z. K. Qin, and D. L. Lin, Phys. Rev. B40, 680 (1989). 


\section{Figure Captions}

Fig.1: Profiles of the total core-shell interaction potential, $V(w)$ plotted as a function of the relative displacement $w$ for three values of the parameter $\mathrm{A}:$ (a) $\mathrm{A}=0.010$,

(b) $\mathrm{A}=0.0187$, and (c) $\mathrm{A}=0.05$.

Fig.2: Variation of the free energy $F_{v} v s$ the reduced temperature $t$ for $\lambda=0$ for two different regimes: (a) Ferroelectric regime with $\langle w\rangle \neq 0$, (b) Incommensurate-paraelectric regime with $\langle w\rangle=$ 0 , with $\mathrm{A}=0.010$.

Fig.3: (a) Variation of the reduced ferroelectric phase transition temperature $t_{c}$ vs the potential parameter A, (b) The dependence of the reduced transition temperature $t_{c}$ on the parameter $R$.

Fig.4: The core fluctuation dependence of the order parameter $\langle w\rangle_{T}$ for three values of the parameter A: (a) $\mathrm{A}=0.010$, (b) $\mathrm{A}=0.0187$, and (c) $\mathrm{A}=0.050$.

Fig.5: The core fluctuation dependence of: (a) the core-atom frequency $\Omega$, and (b) the shell-atom frequency $\varpi$, with $\mathrm{A}=0.010$.

Fig.6: Temperature dependence of the core fluctuation $\sigma$ for three values of the parameter A: (a) $\mathrm{A}=0.010$, (b) $\mathrm{A}=0.0187$, and (c) $\mathrm{A}=0.050$.

Fig.7: Evolution of the order parameter $\langle w\rangle_{T}$ vs the reduced temperature for three values of the parameter A: (a) $\mathrm{A}=0.010$, (b) $\mathrm{A}=0.0187$, and (c) $\mathrm{A}=0.050$.

Fig.8: Variation of the ferroelectric phase transition temperature $T_{c} v$ the potential parameter $A$ in the high-temperature regime. 


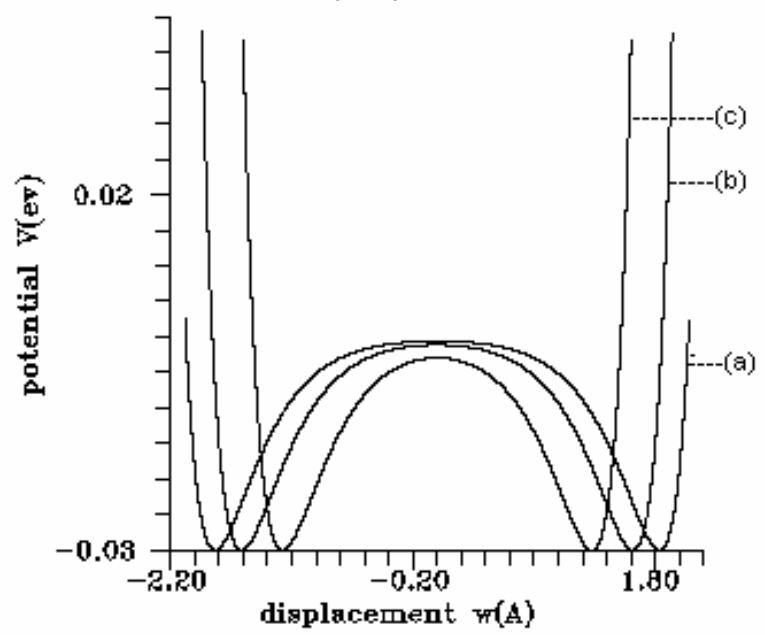

Figure 1

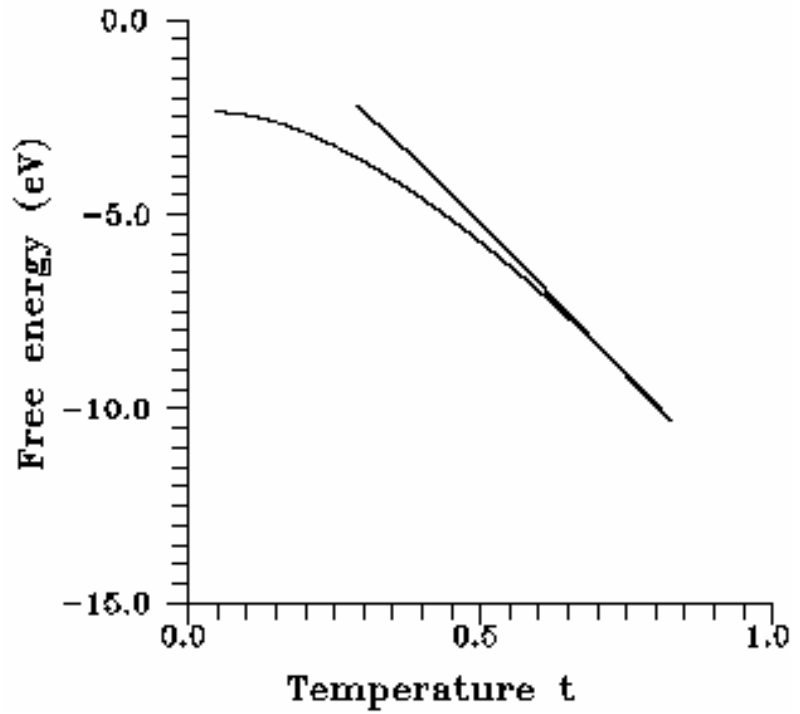

(a)

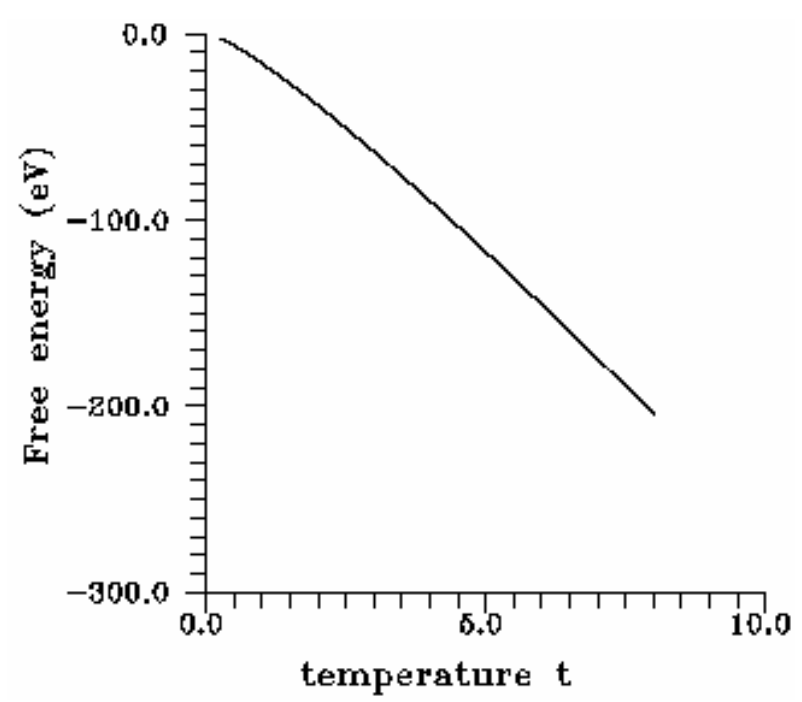

(b)

Figure 2 


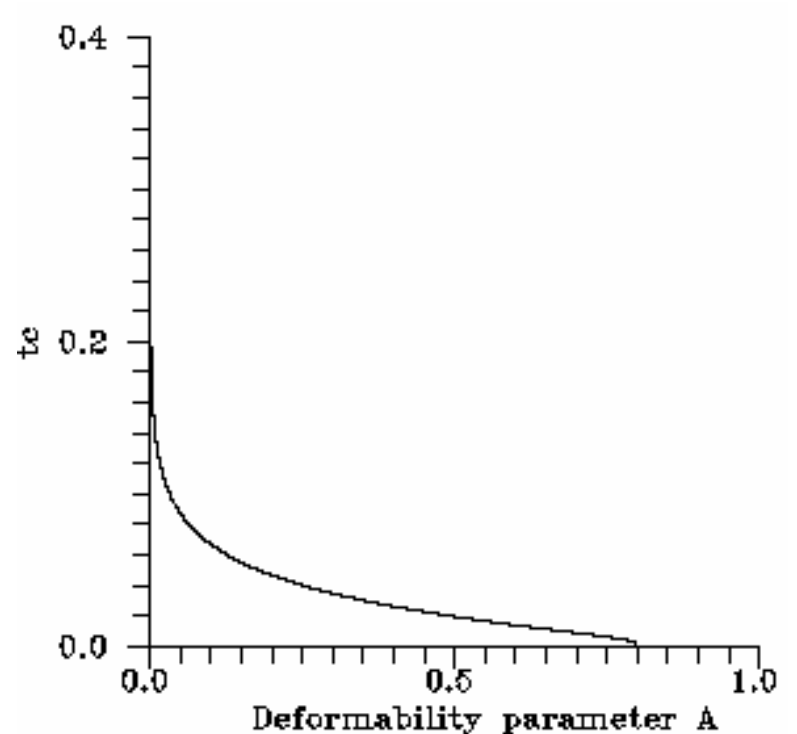

(a)

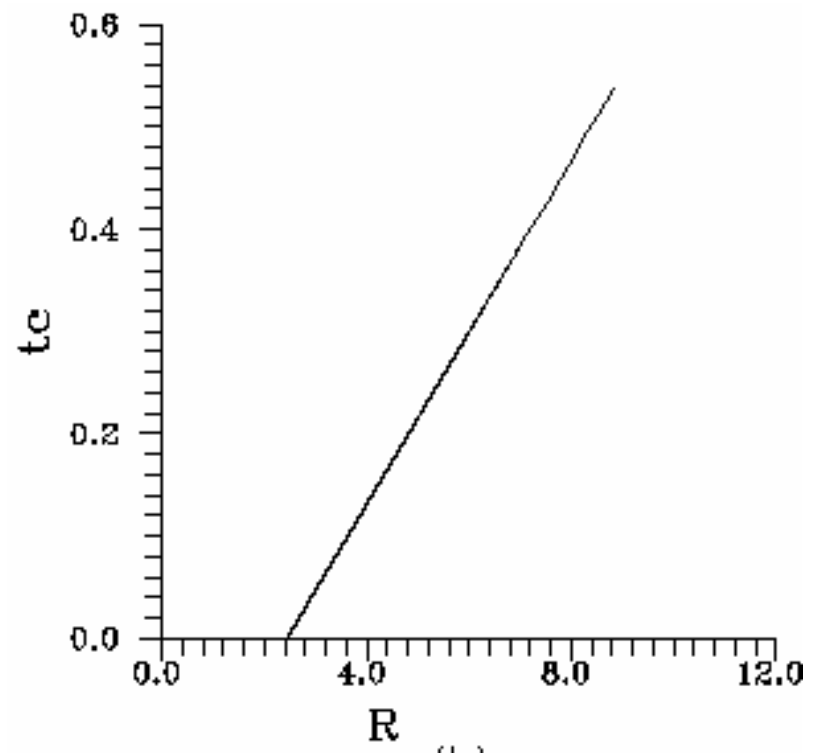

(b)

Figure 3

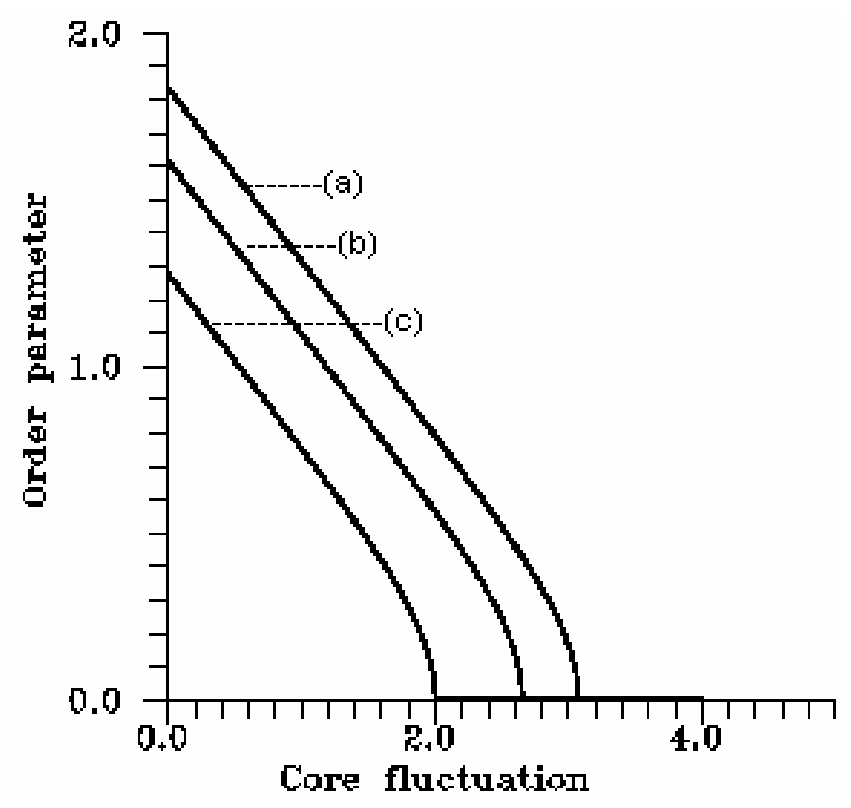

Figure 4 


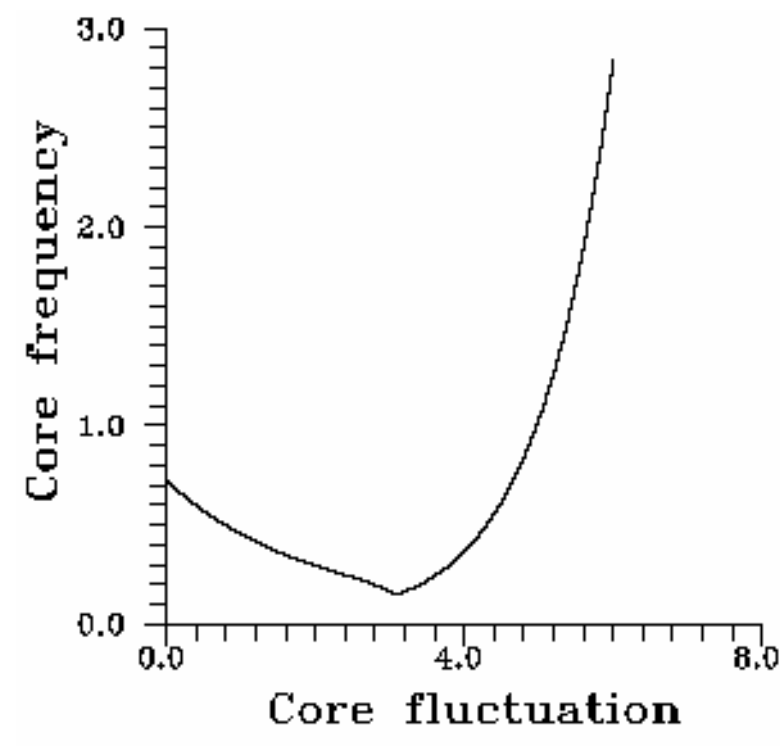

(a)

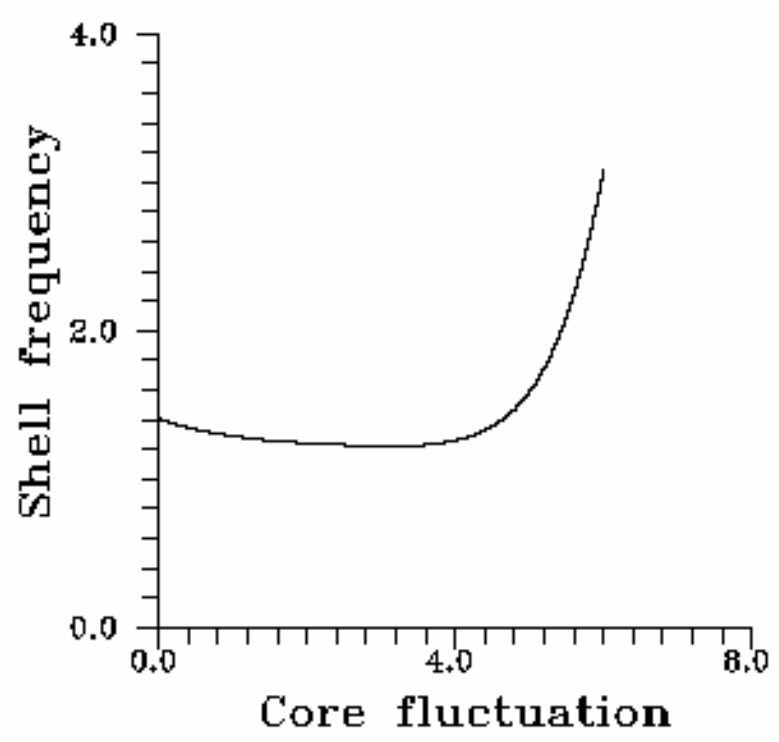

(b)

Figure 5 


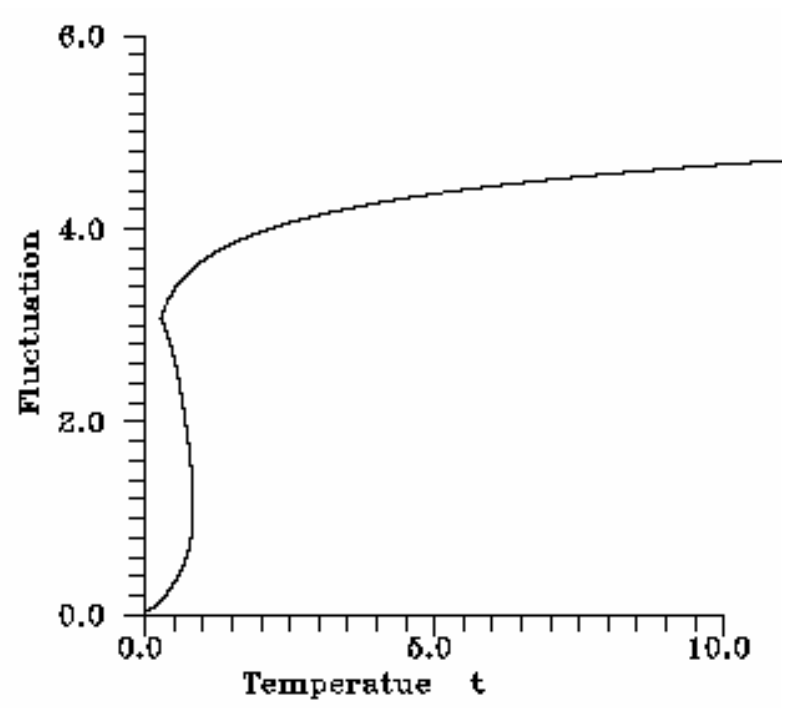

(a)

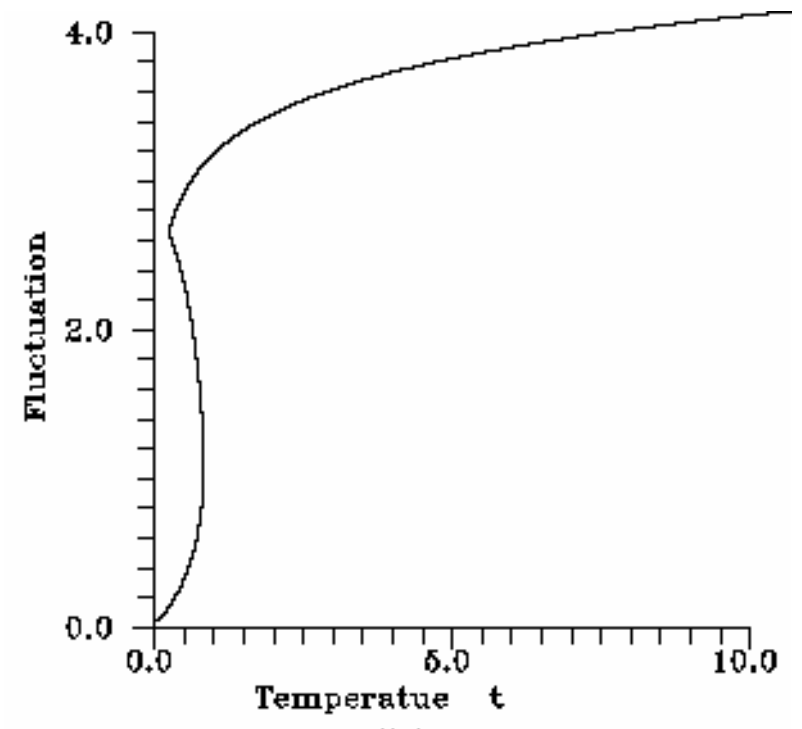

(b)

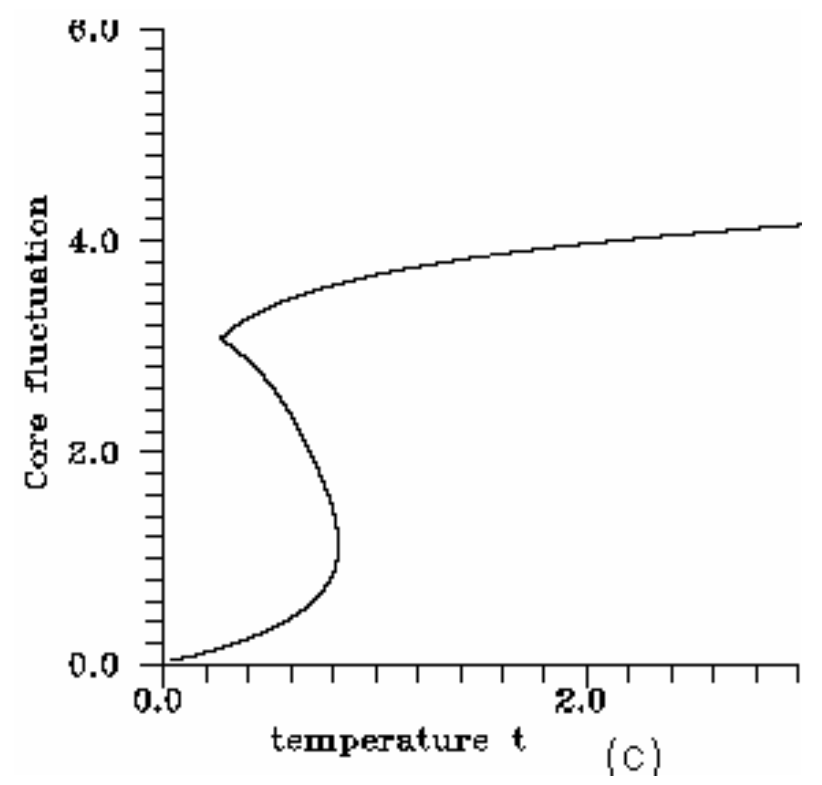

Figure 6 


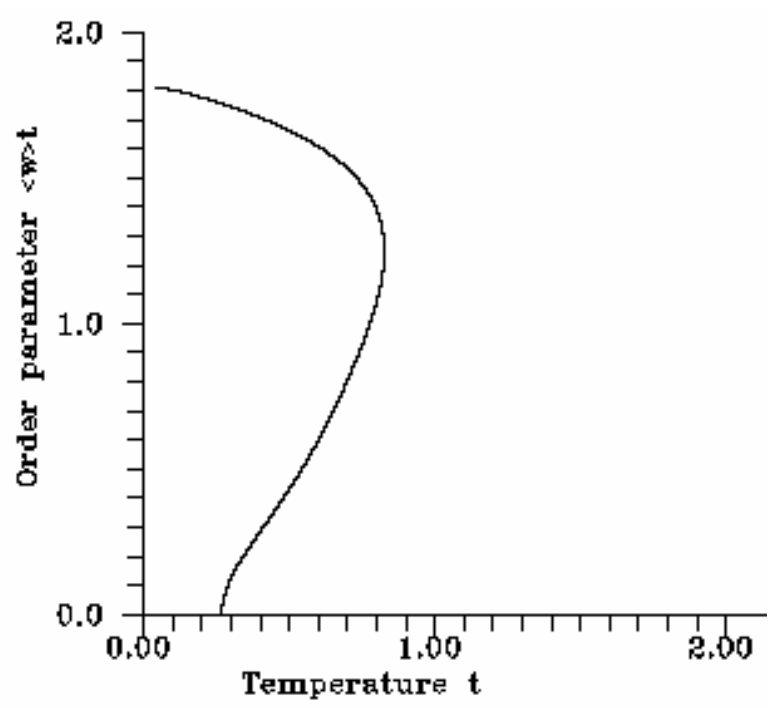

(a)

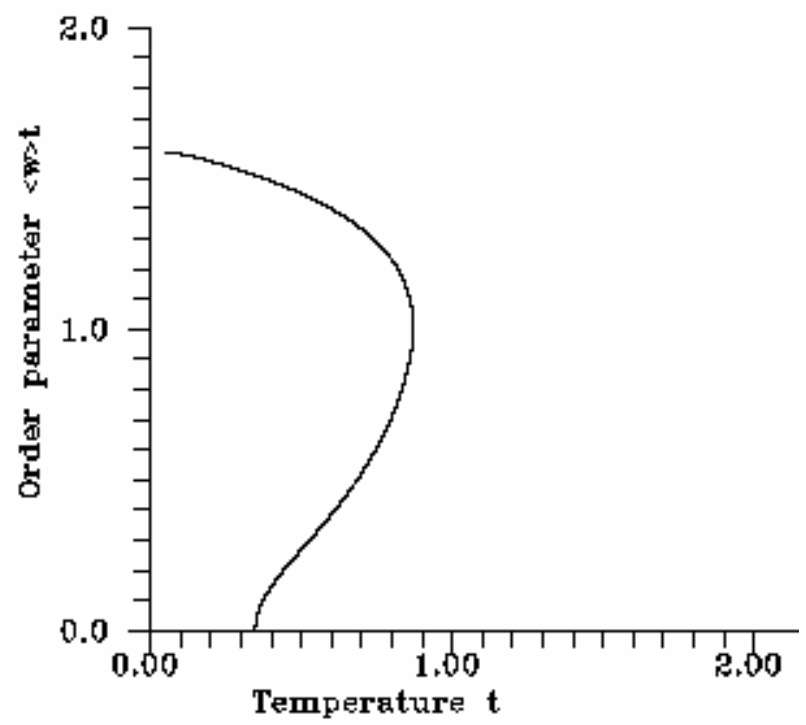

(b)

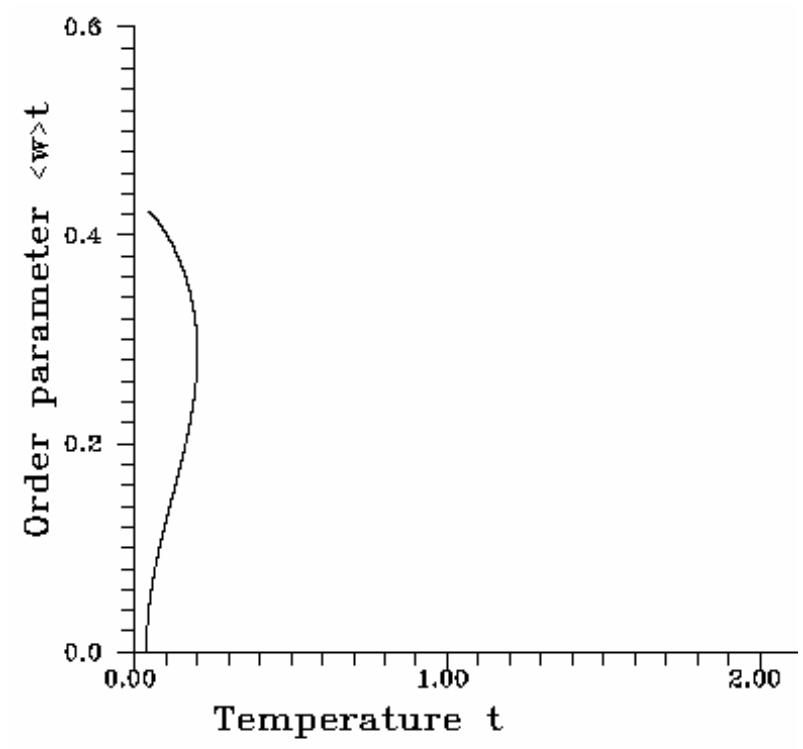

(c)

Figure 7 


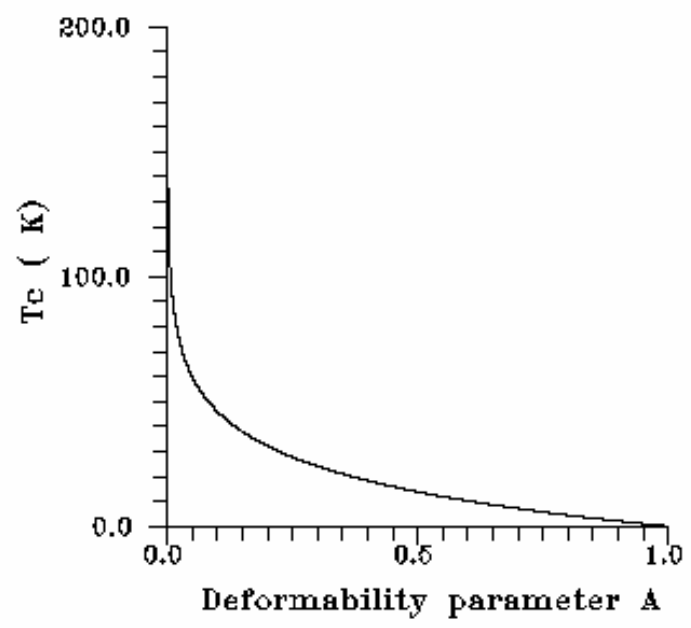

Figure 8 